\title{
Krankenhaus-Directory 2018 - DRG-Krankenhäuser im Vergleich
}

Carina Mostert und Andreas Pritzkau

Elektronisches Zusatzmaterial Die Online-Version dieses Kapitels (https://doi.org/10.1007/978-3-66260487-8_22) enthält Zusatzmaterial, das den Nutzern zur Verfügung steht. 


\section{- Zusammenfassung}

Das Directory deutscher Krankenhäuser bietet eine jährlich aktualisierte Übersicht stationärer Leistungserbringer. Die Darstellung umfasst unter anderem Informationen zur Struktur des vereinbarten Leistungsangebots, zum Grad der Spezialisierung, zur regionalen Marktpositionierung und Wettbewerbssituation sowie Informationen zur Ergebnisqualität nach dem Verfahren Qualitätssicherung mit Routinedaten (QSR). Insgesamt finden mehr als 1.300 Krankenhäuser Eingang, zu denen eine Budgetvereinbarung für das Jahr 2018 oder QSR-Behandlungsergebnisse vorliegen.

The Directory of German Hospitals provides an annually updated overview of inpatient service providers. It includes information on the structure of the agreed range of services, the degree of specialisation, regional market positioning and competitive situation as well as information on the outcome quality according to QSR, a quality reporting procedure based on routine data of the health care funds. In total, the directory includes more than 1,300 hospitals for which a budget agreement for the year 2018 or QSR treatment outcomes are available.

Das jährliche Directory deutscher Krankenhäuser stellt Eckdaten aus den Aufstellungen der Entgelte und Budgetermittlung (AEB) gemäß Krankenhausentgeltgesetz (KHEntgG) dar. Den Darstellungen liegen Vereinbarungsdaten und nicht die tatsächlich erbrachten Leistungen der jeweiligen Einrichtung zugrunde. Insgesamt finden mehr als 1.300 Krankenhäuser Eingang, zu denen eine Vereinbarung oder QSR-Behandlungsergebnisse vorliegen. Das Krankenhaus-Directory finden Sie unter https://doi.org/10.1007/978-3-662-604878_22.

Die einzelnen Spalten des Directories haben folgende Bedeutung:

\section{- Krankenhausname}

Mit einem * gekennzeichnete Einrichtungen haben nach Abschluss der Vereinbarung 2018 mit einem anderen Krankenhaus fusioniert oder wurden geschlossen.

\section{- Betten}

Jedes Krankenhaus wird nach seiner Bettenzahl klassifiziert und einer von sechs Kategorien zugeordnet. Die verwendeten Symbole bedeuten Folgendes:

$$
\begin{aligned}
<50 & =\text { unter } 50 \text { Betten } \\
<100 & =50 \text { bis unter } 100 \text { Betten } \\
<200 & =100 \text { bis unter } 200 \text { Betten } \\
<500 & =200 \text { bis unter } 500 \text { Betten } \\
<1.000 & =500 \text { bis unter } 1.000 \text { Betten } \\
>1.000 & =\text { über } 1.000 \text { Betten }
\end{aligned}
$$

Die Angaben stammen überwiegend aus dem Jahr 2018, andernfalls aus den Vorjahren.

\section{- " Träger}

In dieser Spalte wird die Trägerschaft des Krankenhauses mit folgenden Abkürzungen geschlüsselt:

ö für öffentlich

fg für freigemeinnützig

$\mathrm{p}$ für privat

\section{- Z-Bax (Zahlbasisfallwert)}

Der Basisfallwert ist der Eurobetrag, der multipliziert mit der Bewertungsrelation den Preis einer DRG-Fallpauschale festlegt. Für die Vergütung der Krankenhausfälle einer laufenden Periode ist der Zahlbasisfallwert maßgeblich, der auch Transferzahlungen aus vergangenen Perioden, sogenannte Erlösausgleiche, berücksichtigt. Außerdem dient der Zahlbasisfallwert auch der sachgerechten Umsetzung unterjährig vereinbarter Gesamtjahreswerte. Der gemittelte Zahlbasisfallwert (Z-Bax) ist ein Indikator für das tatsächlich herrschende Preisniveau des Jahres für Krankenhausleistungen, die nach DRGs vergütet werden. ${ }^{1}$ Der Z-Bax umfasst

1 Der bundesweite Z-Bax steht wochenaktuell unter www.wido.de als Download zur Verfügung. 
alle relevanten $\mathrm{Zu}$ - und Abschlagstatbestände. Deren Vergütung wird ebenfalls je Bewertungsrelation, also analog dem Basisfallwert ausgedrückt (Friedrich et al. 2010). ${ }^{2}$

In der Spalte für den Basisfallwert ist ein „BE“ zu finden, wenn das gesamte Krankenhaus 2018 keine DRG-Entgelte vereinbart hat, z. B. auf Basis der Vereinbarung zur Bestimmung von Besonderen Einrichtungen 2018, und es somit als Ganzes von der Anwendung der DRG-Fallpauschalen ausgenommen ist.

\section{- Casemix}

Der Casemix ist die Summe aller Bewertungsrelationen einer Einrichtung. Jedes Krankenhaus wird anhand des vereinbarten Casemix klassifiziert und einer von sechs Kategorien zugeordnet. Die verwendeten Symbole bedeuten Folgendes:

$$
\begin{aligned}
<1.000= & \text { unter } 1.000 \text { Bewertungs- } \\
& \text { relationen } \\
<5.000= & 1.000 \text { bis unter } 5.000 \\
& \text { Bewertungsrelationen } \\
<10.000= & 5.000 \text { bis unter } 10.000 \\
& \text { Bewertungsrelationen } \\
<20.000= & 10.000 \text { bis unter } 20.000 \\
& \text { Bewertungsrelationen } \\
<50.000= & 20.000 \text { bis unter 50.000 } \\
& \text { Bewertungsrelationen }
\end{aligned}
$$

2 Alle fallbezogenen Zuschläge werden bei Anrechnung im Z-Bax durch den vereinbarten CMI des Hauses dividiert. Die berücksichtigten $\mathrm{Zu}$ - und Abschläge lauten z. Zt.: Zuschlag Ausbildung, Investitionszuschlag, Zu- und Abschlag Qualität, Sicherstellungszuschlag, Zuschlag für Vorhaltekosten Besonderer Einrichtungen, Abschlag Tariferhöhung, Abschlag für Mehrleistungen, Abschlag für Nichtteilnahme am DTA, Zu- und Abschlag für die (Nicht-) Teilnahme an der Notfallversorgung, Ausgleiche, Konvergenz Besondere Einrichtungen, Pflegesonderprogramm, Hygieneförderprogramm, Mehrkosten G-BA, Fixkostendegressionsabschlag, Versorgungszuschlag, Pflegezuschlag sowie Zuschläge für Klinische Sektionen, einrichtungsübergreifende Fehlermeldesysteme und bessere Vereinbarkeit von Familie und Beruf. Unberücksichtigt bleiben Zuschläge für Begleitpersonen, Zentren und Schwerpunkte und Telematik.

$$
\begin{aligned}
>50.000= & \text { über } 50.000 \text { Bewertungs- } \\
& \text { relationen }
\end{aligned}
$$

\section{- CMI (Casemix-Index)}

Der Casemix-Index (CMI) beschreibt die mittlere Fallschwere eines Krankenhauses. Er berechnet sich aus dem Quotienten des Casemix (Summe aller Bewertungsrelationen eines Krankenhauses) und der Gesamtzahl der über DRGs abgerechneten Fälle eines Krankenhauses. Der hier ausgewiesene CMI enthält keine teilstationären DRGs.

\section{- Abw. CMI Land}

Für jede Einrichtung wird der individuelle CMI mit dem entsprechenden Landeswert verglichen. Die Abweichungen sind mit folgenden Symbolen gekennzeichnet:

$$
\begin{aligned}
+++= & \text { Abweichung vom Landeswert } \\
& \text { von über } 20 \% \\
++= & \text { Abweichung vom Landeswert } \\
& \text { von } 10 \% \text { bis unter } 20 \% \\
+= & \text { Abweichung vom Landeswert } \\
& \text { von } 0 \% \text { bis unter } 10 \% \\
-= & \text { Abweichung vom Landeswert } \\
& \text { von } 0 \% \text { bis über }-10 \% \\
--= & \text { Abweichung vom Landeswert } \\
& \text { von }-10 \% \text { bis über }-20 \% \\
---= & \text { Abweichung vom Landeswert } \\
& \text { von unter }-20 \%
\end{aligned}
$$
$---=$ Abweichung vom Landeswert von unter $-20 \%$

\section{- Vereinbarter Spezialisierungsgrad} im DRG-Bereich (Gini-Koeffizient)

Die Werte beschreiben den Grad der Spezialisierung für DRG-Leistungen des jeweiligen Krankenhauses anhand des Gini-Koeffizienten. Die Ermittlung erfolgt auf der Ebene BasisDRG (ADRG). Der Gini-Koeffizient ist eine Maßzahl für die (Un)gleichverteilung innerhalb einer Grundgesamtheit. Sind die Leistungen eines Krankenhauses über alle Basis-DRGs gleich verteilt, liegt keine Spezialisierung vor. Verteilen sich die Fälle auf nur wenige BasisDRGs und ist die Verteilung somit sehr ungleich, kann das Krankenhaus als spezialisiert 


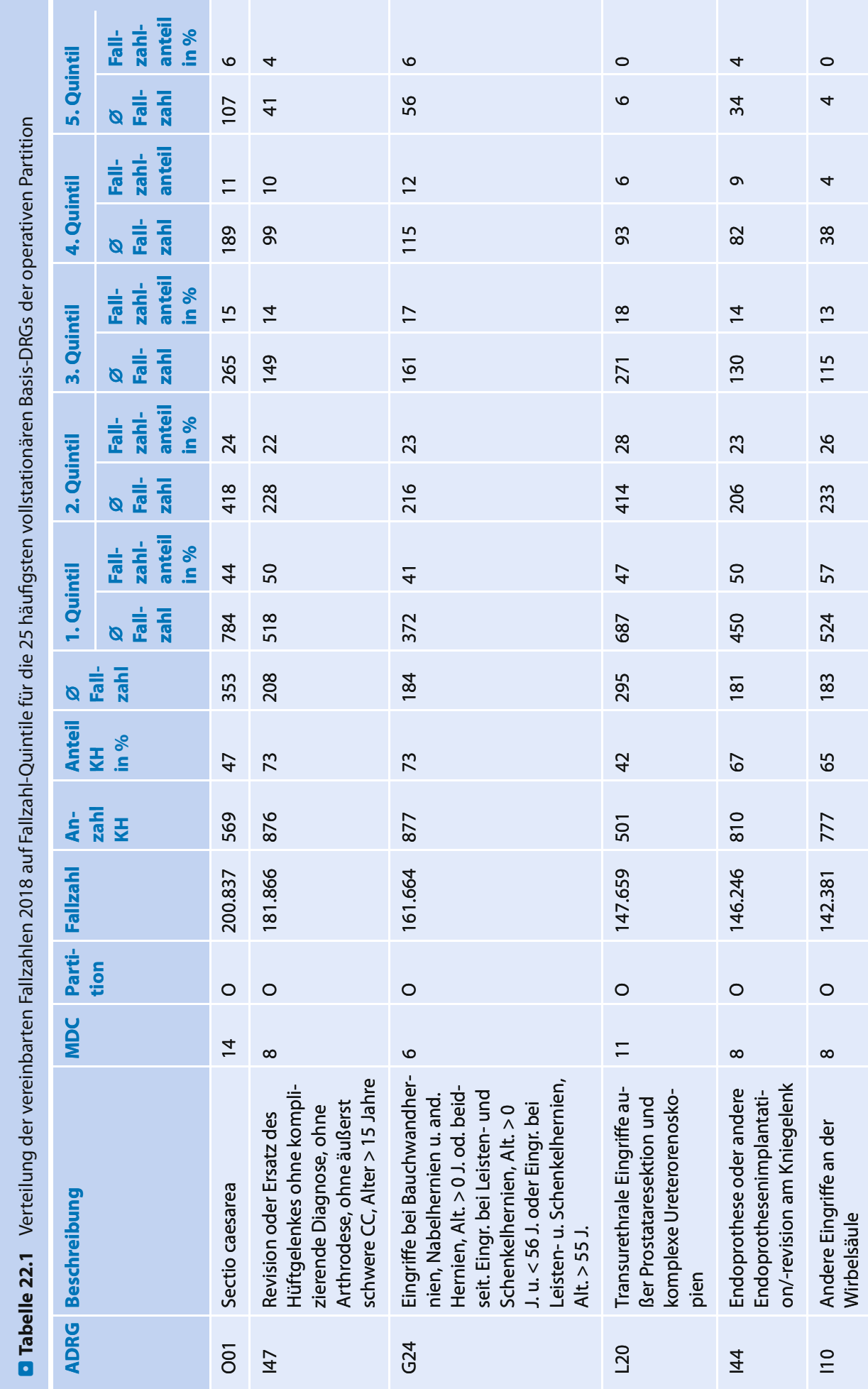




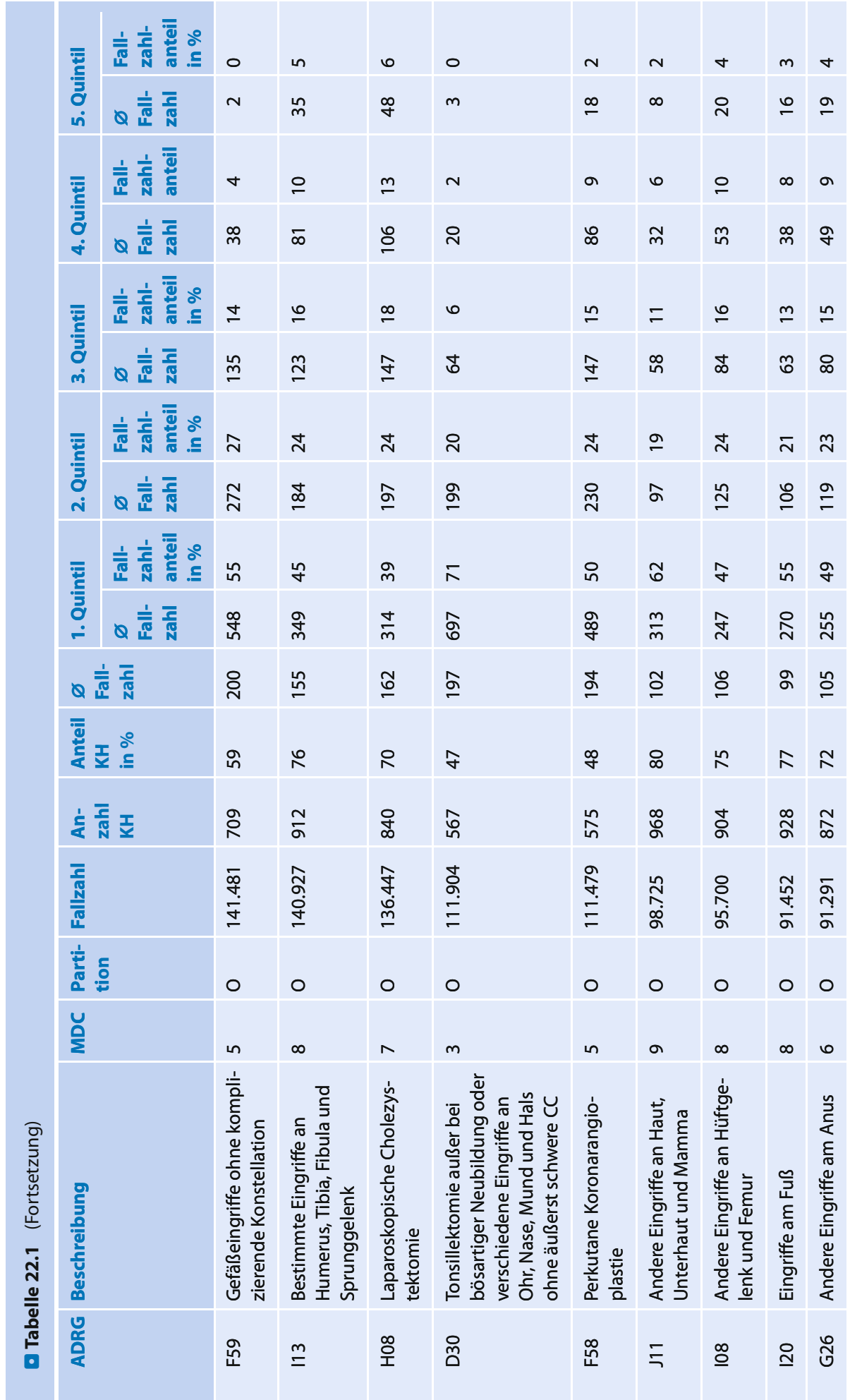




\begin{tabular}{|c|c|c|c|c|c|c|}
\hline \multirow{2}{*}{$\begin{array}{l}\bar{z} \\
\frac{\bar{z}}{3} \\
\text { ○े } \\
\text { ம }\end{array}$} & 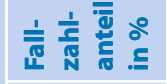 & $\nabla$ & $N$ & in & - & $N$ \\
\hline & $Q \stackrel{\frac{1}{\bar{N}}}{\frac{5}{\mathrm{~N}}}$ & $\stackrel{a}{-}$ & $\stackrel{m}{\square}$ & $\stackrel{\sim}{\sim}$ & $\nabla$ & $a$ \\
\hline \multirow{2}{*}{$\begin{array}{l}\overline{\bar{z}} \\
\bar{c} \\
\bar{z} \\
\dot{f} \\
\dot{f}\end{array}$} & 方衣突 & $=$ & $ㅇ$ & $\simeq$ & $m$ & 0 \\
\hline & Q 䛜 & $\bar{n}$ & $\stackrel{\operatorname{Ln}}{\wedge}$ & 8 & $\stackrel{\sim}{N}$ & $\bar{m}$ \\
\hline \multirow{2}{*}{ 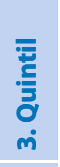 } & 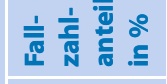 & $\approx$ & $\stackrel{\infty}{\sim}$ & $\stackrel{\infty}{-}$ & $a$ & $\stackrel{m}{-}$ \\
\hline & $Q \frac{1}{\overline{\bar{\Gamma}}} \overline{\frac{5}{\mathrm{~N}}}$ & হ & શิ & $\widehat{\infty}$ & $\mathbb{N}$ & ஜ \\
\hline \multirow{2}{*}{$\begin{array}{l}\overline{\bar{c}} \\
\stackrel{\bar{c}}{\bar{z}} \\
\text { ì } \\
\text { i }\end{array}$} & 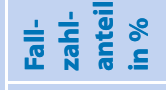 & $\stackrel{ \pm}{\sim}$ & $\stackrel{\llcorner}{\sim}$ & $\stackrel{\sim}{\sim}$ & $\stackrel{\Delta}{\sim}$ & $\stackrel{+}{\sim}$ \\
\hline & $Q \stackrel{\frac{1}{\pi}}{\frac{2}{N}}$ & $\stackrel{n}{=}$ & $\stackrel{\infty}{\infty}$ & $\cong$ & $\stackrel{\infty}{0}$ & $\stackrel{\stackrel{d}{I}}{ }$ \\
\hline \multirow{2}{*}{$\begin{array}{l}\overline{\bar{c}} \\
\stackrel{\bar{c}}{\bar{z}} \\
\stackrel{\circ}{\circ} \\
\end{array}$} & 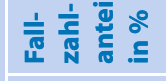 & F & 年 & $\bar{\gamma}$ & $\tilde{0}$ & i̊ \\
\hline & $Q \stackrel{\overline{\bar{\pi}}}{\frac{\mathrm{C}}{\mathrm{N}}}$ & $\frac{\circ}{i}$ & $\underset{\mathrm{m}}{\mathrm{m}}$ & ○े & જे & $\stackrel{\text { o }}{\sim}$ \\
\hline \multicolumn{2}{|c|}{$Q$ 产 } & ณ & $\stackrel{\mathscr{O}}{ \pm}$ & $\stackrel{\infty}{\sigma}$ & $\stackrel{\infty}{\stackrel{n}{n}}$ & $\stackrel{m}{\circ}$ \\
\hline \multicolumn{2}{|c|}{ 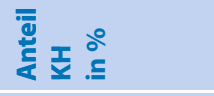 } & $\stackrel{n}{\wedge}$ & $\stackrel{\infty}{+}$ & $\bar{\lambda}$ & $\stackrel{m}{q}$ & $\hat{0}$ \\
\hline \multicolumn{2}{|c|}{ 安蛋 } & \&̊ & 品 & 岗 & $\frac{7}{\text { in }}$ & $\stackrel{9}{1}$ \\
\hline \multicolumn{2}{|l|}{ 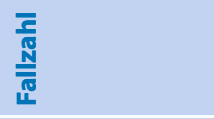 } & 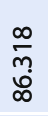 & $\begin{array}{l}\overline{0} \\
\text { in } \\
\text { ฌ }\end{array}$ & \begin{tabular}{l}
\multirow{N}{\hat{b}}{} \\
$\stackrel{\infty}{\infty}$
\end{tabular} & $\frac{\stackrel{0}{n}}{m}$ & $\begin{array}{l}\frac{n}{j} \\
\text { ¿̊. }\end{array}$ \\
\hline \multicolumn{2}{|c|}{ 新 } & 0 & 0 & 0 & 0 & 0 \\
\hline \multicolumn{2}{|l|}{ 产 } & $\infty$ & in & 0 & $m$ & $\infty$ \\
\hline
\end{tabular}

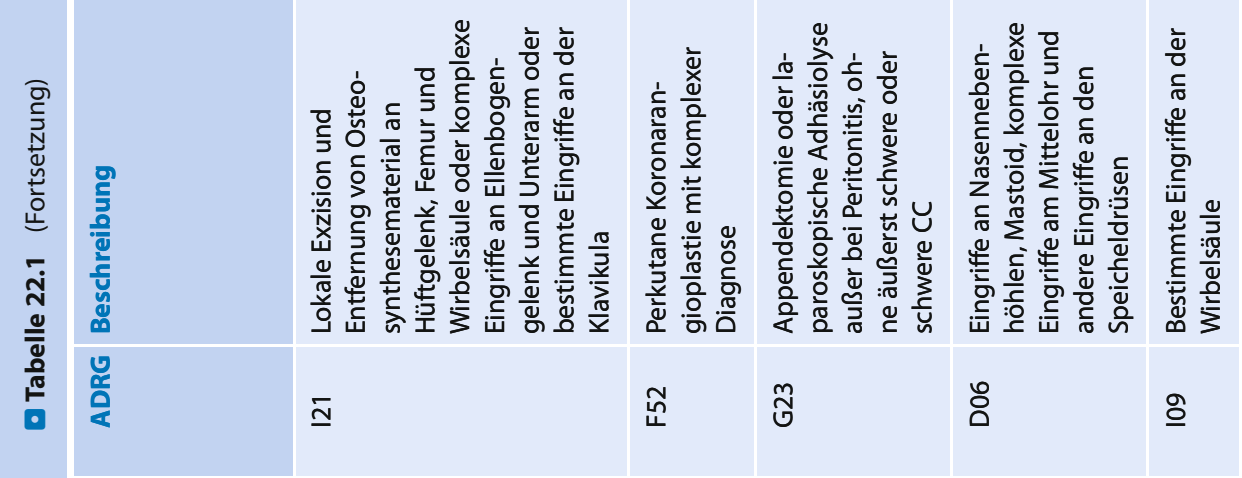




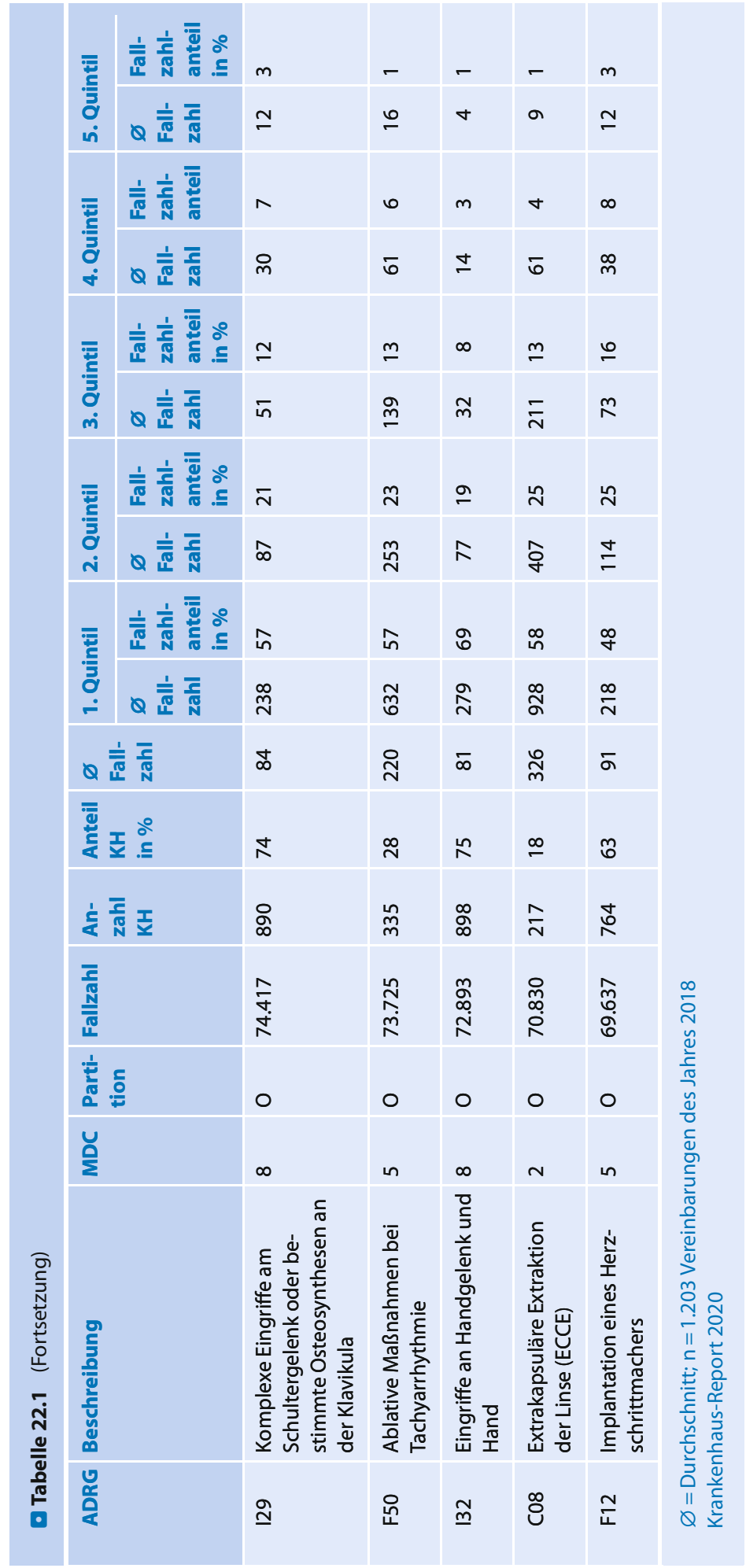




\begin{tabular}{|c|c|c|c|c|c|c|c|c|c|c|}
\hline & 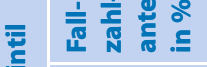 & - & in & 0 & $N$ & $m$ & $m$ & 0 & $N$ & - \\
\hline & 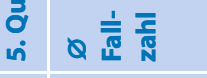 & o & $\stackrel{\circ}{\sim}$ & $\stackrel{m}{\sim}$ & ๙े & i̊n & $\hat{m}$ & $\bar{n}$ & N & $r$ \\
\hline & 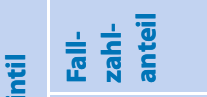 & $=$ & $=$ & $=$ & $\infty$ & $\simeq$ & $=$ & $N$ & $a$ & N \\
\hline & 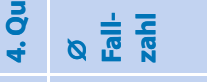 & $\hat{m}$ & గొ & శิ & $\stackrel{\vec{m}}{m}$ & $\stackrel{\ddagger}{\infty}$ & $\stackrel{\wp}{\dot{q}}$ & $\stackrel{n}{0}$ & $\stackrel{\Xi}{=}$ & $\stackrel{\sim}{\sim}$ \\
\hline & $\overline{\bar{z}}$ & $\stackrel{\infty}{-}$ & $\stackrel{\circ}{-}$ & $\stackrel{\circ}{\circ}$ & $\stackrel{n}{n}$ & $\stackrel{\infty}{-}$ & $=$ & $m$ & $\stackrel{\circ}{\sim}$ & $\circ$ \\
\hline & $\begin{array}{l}\text { Oे } \\
\dot{m}\end{array}$ & స్రి & $\bar{\infty}$ & ठે & $\stackrel{\text { I }}{\sim}$ & సે & సิ & 拿 & ஓ् & 0 \\
\hline & $\overline{\bar{z}} \overline{\bar{z}}$ & $\stackrel{\sim}{\sim}$ & $\stackrel{ \pm}{\sim}$ & $\stackrel{ \pm}{\sim}$ & $\stackrel{+}{\sim}$ & $\stackrel{\mathscr{N}}{\sim}$ & $\stackrel{\stackrel{\sim}{ }}{ }$ & $\stackrel{\infty}{\sim}$ & $\stackrel{\stackrel{\sim}{ }}{ }$ & $\stackrel{ \pm}{\sim}$ \\
\hline & $\begin{array}{l}\bar{z} \\
\text { i }\end{array}$ & \&ু & $\stackrel{\widehat{N}}{\stackrel{N}{\sim}}$ & $\stackrel{\swarrow}{\infty}$ & $\bar{q}$ & बे & ₹ & $\stackrel{\substack{\stackrel{\infty}{N} \\
i}}{ }$ & m & I \\
\hline & 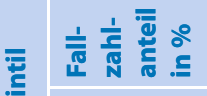 & 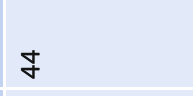 & $\mathscr{q}$ & $\mathcal{F}$ & กิ & F & \& & 6 & $\stackrel{\infty}{\sigma}$ & 6 \\
\hline & 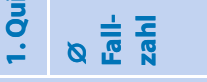 & $\stackrel{m}{n}$ & 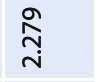 & $\stackrel{\bar{\infty}}{\stackrel{\leftrightarrow}{n}}$ & ڤે & $\tilde{6}$ & $\overline{\widehat{\sigma}}$ & बे & จิธ & $\stackrel{\circ}{\circ}$ \\
\hline & $Q \frac{1}{\overline{\bar{L}}} \frac{\overline{\mathrm{N}}}{\mathrm{N}}$ & 今ั & $\underset{g}{\stackrel{g}{0}}$ & $\bar{n}$ & 商 & $\frac{\infty}{m}$ & $\stackrel{n}{\curvearrowright}$ & $\stackrel{\widetilde{N}}{\text { Oे }}$ & $\stackrel{\circ}{\sim}$ & $\stackrel{n}{\sim}$ \\
\hline & 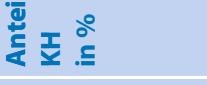 & Ф & in & $\stackrel{\infty}{+}$ & ळ & $\infty$ & $\widetilde{\infty}$ & $=$ & $\infty$ & 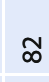 \\
\hline & 安蛋 & $\stackrel{\circ}{\circ}$ & 8 & N & م્a & \&̆ & œ & $\stackrel{m}{m}$ & ఫ̊ & $\stackrel{m}{\circ}$ \\
\hline & 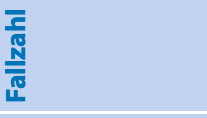 & $\begin{array}{l}\stackrel{\aleph}{0} \\
\text { ò } \\
\stackrel{R}{0}\end{array}$ & 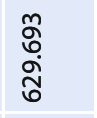 & 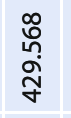 & 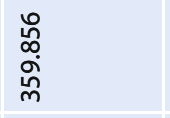 & 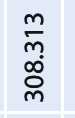 & 莡 & $\begin{array}{l}\text { ஸे } \\
\stackrel{0}{N} \\
\text { }\end{array}$ & $\begin{array}{l}\stackrel{0}{0} \\
\text { ஸे } \\
\text { N }\end{array}$ & $\begin{array}{l}\stackrel{ \pm}{\infty} \\
\stackrel{\sim}{\bar{D}}\end{array}$ \\
\hline & 㰴 & $\Sigma$ & $\Sigma$ & $\Sigma$ & $\Sigma$ & $\Sigma$ & $\Sigma$ & $\Sigma$ & $\Sigma$ & $\Sigma$ \\
\hline & 产 & $\bullet$ & $\stackrel{n}{\llcorner}$ & \pm & $\infty$ & in & $\sigma$ & $=$ & in & - \\
\hline & 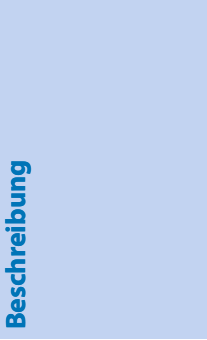 & 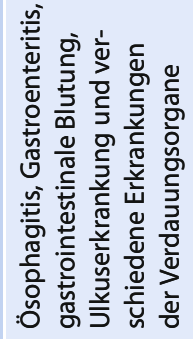 & 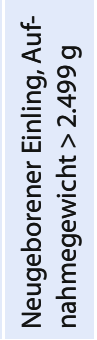 & 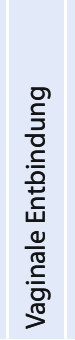 & 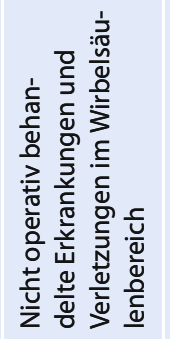 & 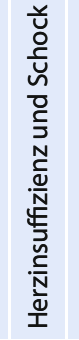 & 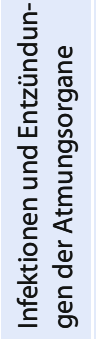 & 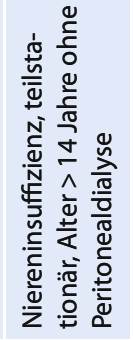 & 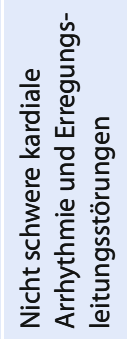 & $\begin{array}{l}\frac{0}{x} \\
\frac{0}{0} \\
\frac{0}{4}\end{array}$ \\
\hline & & ᄋ్ర & ᄋ̊ & ○ & $\stackrel{\infty}{\stackrel{\infty}{0}}$ & ర్రు & 욜 & $\stackrel{8}{9}$ & 疋 & 冬 \\
\hline
\end{tabular}




\begin{tabular}{|c|c|c|c|c|c|c|c|c|c|c|c|c|}
\hline \multirow{2}{*}{ 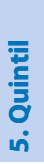 } & 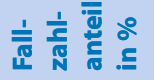 & - & - & $m$ & $m$ & $\sim$ & - & $\sim$ & $m$ & $\sim$ & - & $\sim$ \\
\hline & $Q \stackrel{\overline{\bar{N}}}{\overline{\mathrm{N}}}$ & $\stackrel{\text { ๓ }}{2}$ & $\mp$ & $\ddot{\sim}$ & ৯ & ๗ & 으 & $\stackrel{2}{-}$ & 유 & $\approx$ & 0 & $\simeq$ \\
\hline \multirow{2}{*}{ 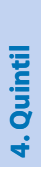 } & 言 $\frac{1}{\frac{2}{N}}$ & $\lambda$ & a & $\simeq$ & 으 & $a$ & $\checkmark$ & $\mp$ & 으 & $a$ & $m$ & $\infty$ \\
\hline & $Q \stackrel{\frac{1}{\bar{N}}}{\frac{2}{N}}$ & হ & 옴 & $\stackrel{0}{=}$ & ลิ & $\infty$ & $\widehat{m}$ & ส & $\infty$ & $\stackrel{m}{\wedge}$ & $\stackrel{\sim}{\sim}$ & 6 \\
\hline \multirow{2}{*}{$\begin{array}{l}\bar{z} \\
\text { m. } \\
\text { m. } \\
\text { m. }\end{array}$} & 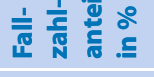 & $\stackrel{m}{-}$ & $\stackrel{6}{-}$ & $\stackrel{\infty}{-}$ & $\stackrel{0}{-}$ & $\stackrel{0}{-}$ & $a$ & $\stackrel{\infty}{-}$ & $\underline{-}$ & $\stackrel{6}{-}$ & 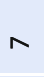 & $\stackrel{\text { n }}{\leftarrow}$ \\
\hline & $Q \stackrel{\frac{1}{\sqrt{N}}}{\bar{N}}$ & 守 & ิㅗ & 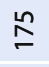 & ํㅡ & J & $\infty$ & I & $\stackrel{m}{\underline{m}}$ & 음 & กิ & 음 \\
\hline
\end{tabular}

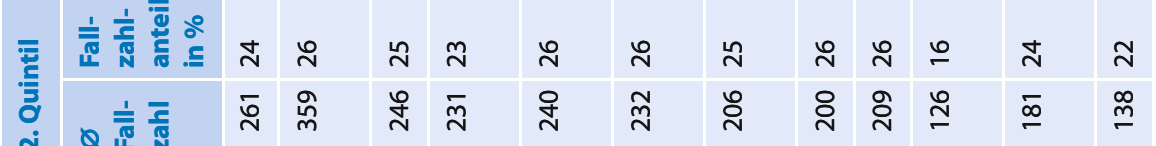

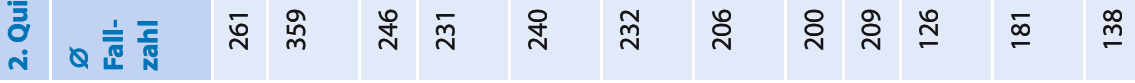

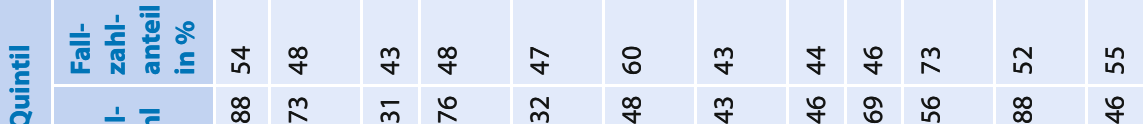

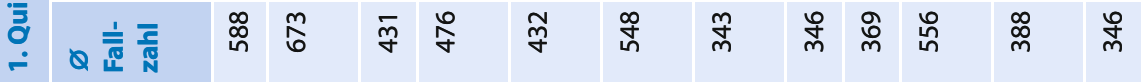

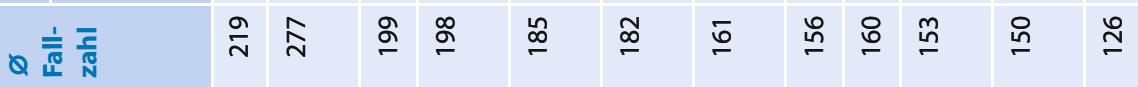

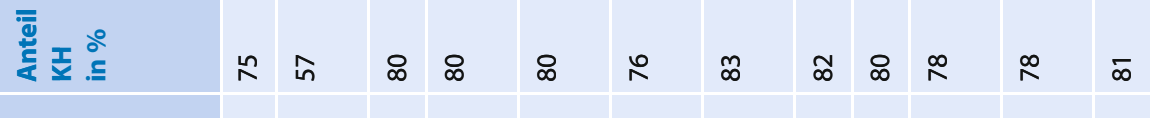

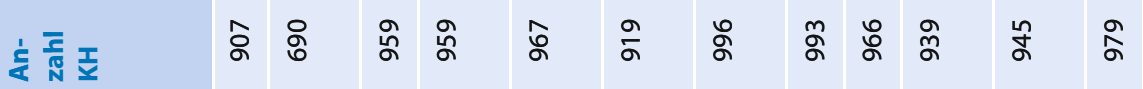

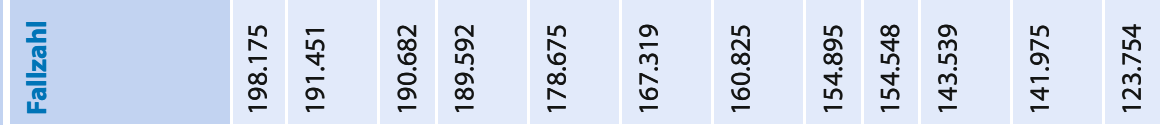

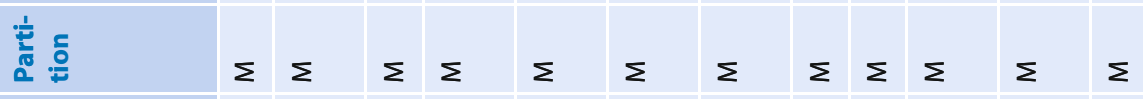
跑

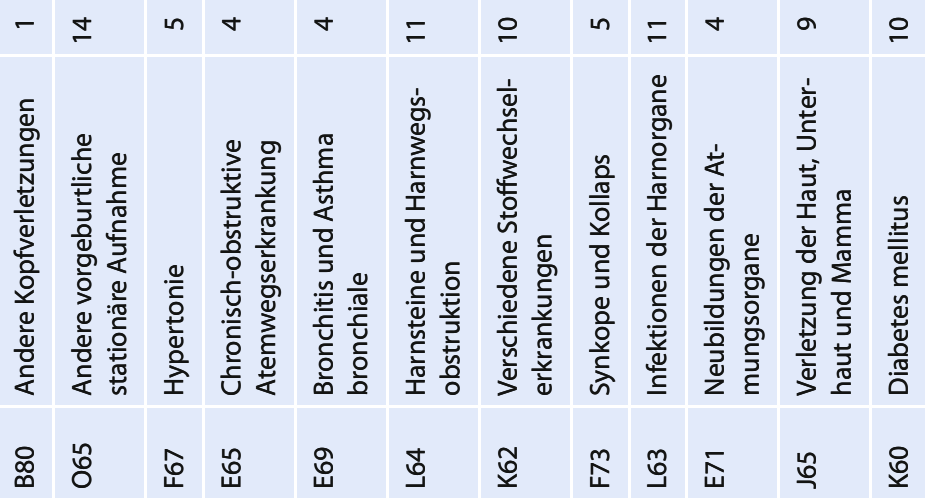




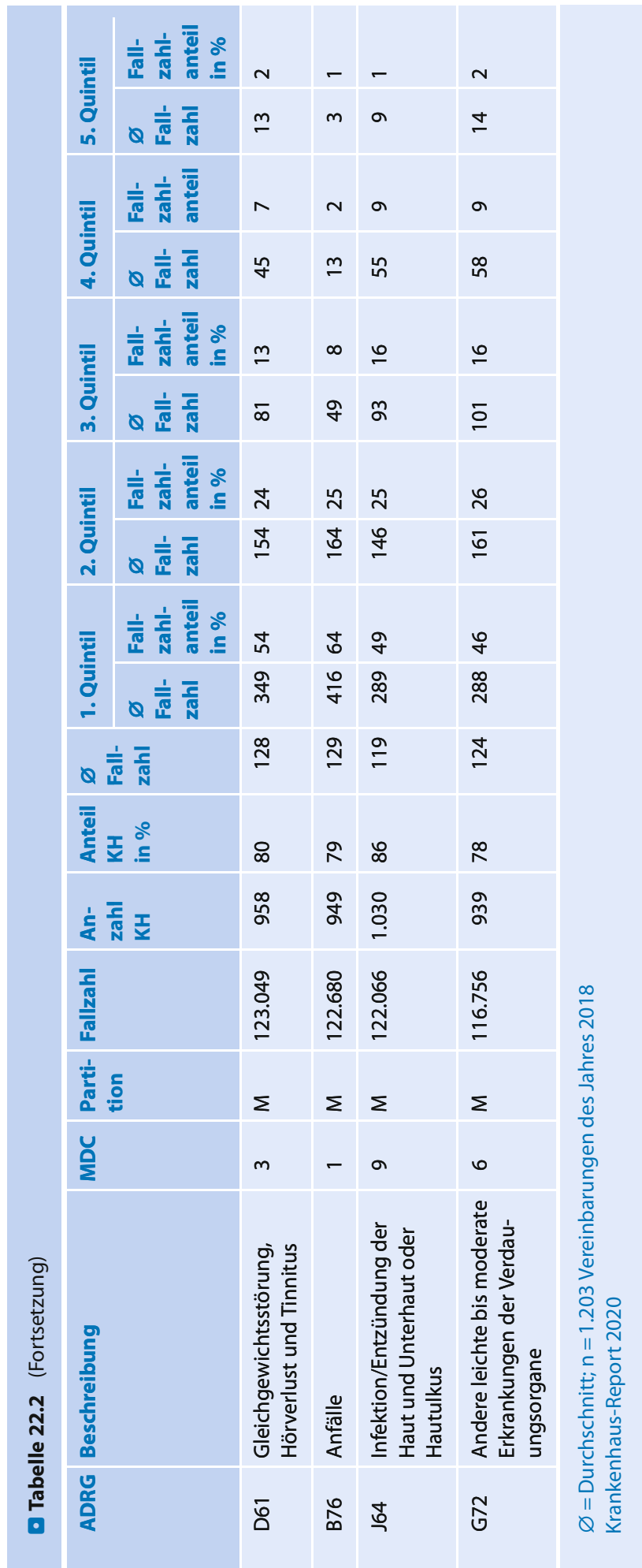


gelten. Ein Gini-Koeffizient von 1 resultierte aus einer maximalen Spezialisierung auf nur eine Leistung, ein Wert von 0 entspräche einer identischen Fallzahl in allen Basis-DRGs.

Aus dem Grad der Spezialisierung der Krankenhäuser lassen sich nur wenige Rückschlüsse auf die Zentralisierung der Leistungserbringung ziehen. Die - Tab. 22.1 und 22.2 illustrieren die Verteilung der Fallzahlen je vollstationäre Basis-DRG (s.u.) der operativen bzw. der medizinischen Partition auf die vorliegenden Vereinbarungen. Langversionen der Tabellen mit allen vollstationären BasisDRGs sind als elektronisches Zusatzmaterial unter https://doi.org./10.1007/978-3-66260487-8_22 (Tab 22.a und 22.b) zu finden. Die Darstellung erfolgt nach Fallzahlquintilen. Die Spalten zum ersten Quintil geben z. B. darüber Auskunft, welchen Anteil die 20\% der Krankenhäuser mit den größten Fallzahlen am Gesamtaufkommen haben. Die Spalten zum fünften Quintil geben u. a. Hinweise, in welchen Basis-DRGs die $20 \%$ der Krankenhäuser mit den geringsten Fallzahlen die entsprechende Leistung nur sehr selten erbringen. Die Darstellung beschränkt sich auf die jeweils 25 fallzahlstärksten Basis-DRGs.

\section{- Leistungsdichte Basis-DRGs}

Es wird jeweils angegeben, wie viele BasisDRGs (ADRGs) jeweils 25\%, $50 \%$ und $75 \%$ aller Leistungen eines Hauses ausmachen. Basis-DRGs stellen eine Obergruppe für eine oder mehrere DRGs dar, die durch die gleichen Diagnosen- und/oder Prozedurencodes definiert sind. DRGs innerhalb einer Basis-DRG unterscheiden sich in ihrem Ressourcenverbrauch bzw. ihrem Schweregrad. In der G-DRG Version 2018 gibt es 563 Basis-DRGs, davon drei nicht bewertete Fehler-DRGs und eine teilstationäre DRG.

\section{- TOP 3 MDC}

In einer weiteren Annäherung an das DRGLeistungsspektrum eines Hauses werden die fünf jeweils stärksten Hauptdiagnosegruppen (MDCs; Major Diagnostic Categories) mit ihrer Nummer sowie dem jeweiligen Prozentan- teil an sämtlichen DRG-Leistungen dokumentiert. Die Nummern der MDCs bedeuten Folgendes:

-1 Pre-MDC

1 Krankheiten und Störungen des Nervensystems

2 Krankheiten und Störungen des Auges

3 Krankheiten und Störungen im HNO-Bereich

4 Krankheiten und Störungen der Atmungsorgane

5 Krankheiten und Störungen des Kreislaufsystems

6 Krankheiten und Störungen der Verdauungsorgane

7 Krankheiten und Störungen am hepatobiliären System und Pankreas

8 Krankheiten und Störungen am Muskel-Skelett-System und Bindegewebe

9 Krankheiten und Störungen an Haut, Unterhaut und Mamma

10 Endokrine, Ernährungs- und Stoffwechselkrankheiten

11 Krankheiten und Störungen der Harnorgane

12 Krankheiten und Störungen der männlichen Geschlechtsorgane

13 Krankheiten und Störungen der weiblichen Geschlechtsorgane

14 Schwangerschaft, Geburt und Wochenbett

15 Neugeborene

16 Krankheiten des Blutes, der blutbildenden Organe und des Immunsystems

17 Hämatologische und solide Neubildungen

18 Infektiöse und parasitäre Krankheiten 
19 Psychiatrische Krankheiten und Störungen

20 Alkohol- und Drogengebrauch und alkohol- und drogeninduzierte psychische Störungen

21 Verletzungen, Vergiftungen und toxische Nebenwirkungen von Drogen und Medikamenten

22 Verbrennungen

23 Faktoren, die den Gesundheitszustand beeinflussen und andere Inanspruchnahmen des Gesundheitswesens

\section{Sonstige DRGs}

\section{- Partitionen in \% (Verteilung} über die Partitionen)

Eine MDC kann in drei Partitionen aufgeteilt sein:

- DRGs liegen in der chirurgischen Partition, wenn sie eine Prozedur beinhalten, für die ein OP-Saal erforderlich ist.

- DRGs der anderen Partition beinhalten Prozeduren, die in der Regel diagnostische Maßnahmen abbilden und für die kein OPSaal erforderlich ist.

- DRGs der medizinischen Partition beinhalten keine relevanten Prozeduren.

Die Abkürzungen der Partitionen bedeuten Folgendes:

$$
\begin{aligned}
\mathrm{o} & =\text { operativ } \\
\mathrm{a} & =\text { andere } \\
\mathrm{m} & =\text { medizinisch }
\end{aligned}
$$

\section{- - Budget-Anteile ZE/SE}

Für Leistungen, die mit DRGs noch nicht sachgerecht vergütet werden, können die Vertragspartner individuelle Leistungskomplexe und Entgelte vereinbaren. Dazu gehören im Jahr 2018 u. a. 45 DRGs (davon drei teilstationäre), $\mathrm{zu}$ denen keine sachgerechte Bewertungsrelation durch das InEK ermittelt werden konnte, aber auch Leistungen in besonderen Einrich- tungen und teilstationäre Behandlung. ${ }^{3}$ Die Spalte Budgetanteil SE beschreibt den Anteil solcher tages- oder fallbezogenen Leistungen am Gesamtbudget aus DRGs, Zusatzentgelten und sonstigen Entgelten. Dieser Budgetanteil ist von der Vergütung nach DRGs sowie der Budgetkonvergenz ausgenommen.

Zusatzentgelte können neben DRG-Fallpauschalen sowie tages- und fallbezogenen sonstigen Entgelten zusätzlich abgerechnet werden. Über die 94 vom InEK kalkulierten und bundeseinheitlich vergüteten hinaus können weitere hausindividuelle Zusatzentgelte vereinbart werden.

\section{- Bes. Leist. (B/N/H/P)}

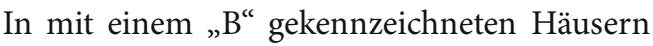
sind Leistungsbereiche vereinbart, die nach der Vereinbarung zur Bestimmung von Besonderen Einrichtungen - VBE 2018 - von der Abrechnung nach DRG-Fallpauschalen und der Budgetkonvergenz ausgenommen sind. „N" markiert Einrichtungen, in denen 2018 Entgelte für neue Untersuchungs- und Behandlungsmethoden nach $₫ 6$ Abs. 2 des Krankenhausentgeltgesetzes (NUB) vereinbart wurden. „H“ kennzeichnet Krankenhäuser, in denen Zusatzentgelte für hochspezialisierte Leistungen nach $₫ 6$ Abs. 2a des Krankenhausentgeltgesetzes vereinbart wurden. „P“ markiert Krankenhäuser mit einer psychiatrischen Fachabteilung.

\section{- Notfall}

In dieser Spalte findet sich ein „N“, sofern für das Krankenhaus im Jahr 2018 ein Abschlag für die Nichtteilnahme an der Notfallversorgung vereinbart wurde.

\section{- AOK-Patientenwege (PKW-km) (Med/oQ)}

Für jede Einrichtung wird auf Basis der AOKKrankenhausfälle mit Abrechnung nach Krankenhausentgeltgesetz (KHEntgG) die maximale PKW-Strecke in $\mathrm{km}$ für die $50 \%$ (in der

3 Die Regelungen finden sich im Detail in $\S 6$ Abs. 1 des Krankenhausentgeltgesetzes. 
Spalte Med für Median) bzw. 75 \% (in der Spalte oQ für oberes Quartil) der AOK-Versicherten mit der kürzesten Fahrtstrecke dargestellt. Als Startpunkt des Patientenwegs gilt der geografische Mittelpunkt des 5-stelligen PLZ-Gebiets des Patientenwohnorts, als Endpunkt die vollständige Adresse des Krankenhauses.

\section{- Vereinbarte regionale DRG-Marktanteile und -konzentration im Umkreis von 10, 20 und 30 km (Marktanteil/HHI)}

Die Spalten beschreiben die regionale Marktund Wettbewerbssituation des jeweiligen Krankenhauses für DRG-Leistungen im Luftlinienumkreis von 10, 20 und $30 \mathrm{~km}$ anhand der Kennzahlen Marktanteil und dem HerfindahlHirschman-Index (HHI).

Der ausgewiesene regionale Marktanteil eines Krankenhauses basiert auf den dort konkret vereinbarten Leistungen. Eine Einrichtung in einer Region mit hoher Krankenhausdichte kann also auch einen relativ hohen Marktanteil aufweisen, sofern es Leistungen erbringt, die in der Region ansonsten selten bzw. in geringem Umfang vereinbart sind.

Der Herfindahl-Hirschman-Index ist eine Kennzahl zur Konzentrationsmessung in einem Markt bzw. in einer Marktregion und spiegelt so die Wettbewerbsintensität wider. Er ist als Summe der quadrierten Markanteile aller Teilnehmer in einer Region definiert und kann die Werte zwischen 0 und 1 annehmen, wobei der Wert 1 als Synonym für eine Monopolstellung keinem Wettbewerb entspricht. Verteilen sich in einer Wettbewerbsregion die Leistungen gleichmäßig auf zwei Anbieter, so haben beide einen Marktanteil von $50 \%$, der quadrierte Marktanteil beträgt jeweils 0,25 und der HHI als Summe der quadrierten Marktanteile ist 0,50. Verteilen sich die Leistungen aber nicht gleichmäßig auf die zwei Anbieter, sondern im Verhältnis $99 \%$ zu $1 \%$, so nimmt der HHI einen Wert in der Nähe von 1 ein und spiegelt so die monopolistische Angebotsstruktur wider.

Um unerwünschte Effekte aus noch nicht geschlossenen Vereinbarungen zu minimieren, basieren die Marktdaten abweichend von den übrigen Werten in der Tabelle aus der Vorjahres-Budgetrunde.

\section{- Infozeile Bundesland}

Die Darstellung ist sortiert nach Bundesländern und dem Namen des Standortes. Für jedes Bundesland werden in einer Zeile die gewichteten Mittelwerte CMI, Anteile der Partitionen an Gesamtfällen, Leistungsdichte Basis-DRG, Top MDC, Budgetanteile von Zusatzentgelten und sonstigen Entgelten sowie die Anzahl der Krankenhäuser mit vereinbarten besonderen Leistungen dargestellt.

\section{- QSR-Behandlungsergebnisse}

Das QSR-Verfahren der AOK ist ein Verfahren zur Qualitätsmessung von Krankenhausbehandlungen. Die Abkürzung QSR steht für „Qualitätssicherung mit Routinedaten“. Im QSR-Verfahren kann durch die konsequente Analyse der Behandlung und des Überlebensstatus bis zu einem Jahr nach der Erstoperation auch die langfristige Behandlungsqualität gemessen werden. Zur Berechnung der Qualitätsindikatoren werden Abrechnungs- bzw. Routinedaten verwendet. Diese werden den Krankenkassen automatisch vom Krankenhaus übermittelt, um die Behandlung eines Patienten in Rechnung $\mathrm{zu}$ stellen, oder liegen der Krankenkasse bereits in den Versichertenstammdaten vor.

Im Krankenhaus-Directory stehen die krankenhausbezogenen Ergebnisse für folgende Leistungsbereiche zur Verfügung: Einsetzen einer Endoprothese oder osteosynthetische Versorgung nach einem hüftgelenknahen Oberschenkelbruch, Einsetzen einer Hüftendoprothese bei Coxarthrose (Hüft-EP), Einsetzen eines künstlichen Kniegelenks bei Gonarthrose (Knie-EP), Gallenblasenentfernung bei Gallensteinen, Blinddarmentfernung, Operation bei gutartiger Prostatavergrößerung, Prostataentfernung bei Prostatakrebs, therapeutische Herzkatheter (PCI) bei $\mathrm{Pa}$ tienten ohne Herzinfarkt, Verschluss einer Leistenhernie und Hüftprothesenwechsel 
(nicht bei Knochenbruch oder Infektion). Das aktuelle Verfahrensjahr 2019 umfasst den Berichtszeitraum 2015 bis 2017 mit 2018 zur 22 Nachbeobachtung der Patienten.

Die klinikbezogenen QSR-Ergebnisse werden auch im AOK-Krankenhausnavigator auf Basis der Weissen Liste frei zugänglich veröffentlicht. ${ }^{4}$

\section{Literatur}

Friedrich J, Leber WD, Wolff J (2010) Basisfallwerte zur Preis- und Produktivitätsentwicklung stationärer Leistungen. In: Klauber J, Geraedts M, Friedrich J (Hrsg) Krankenhaus-Report 2010. Schattauer, Stuttgart, S 122-147 
Open Access Dieses Kapitel wird unter der Creative Commons Namensnennung 4.0 International Lizenz (http:// creativecommons.org/licenses/by/4.0/deed.de) veröffentlicht, welche die Nutzung, Vervielfältigung, Bearbeitung, Verbreitung und Wiedergabe in jeglichem Medium und Format erlaubt, sofern Sie den/die ursprünglichen Autor(en) und die Quelle ordnungsgemäß nennen, einen Link zur Creative Commons Lizenz beifügen und angeben, ob Änderungen vorgenommen wurden.

Die in diesem Kapitel enthaltenen Bilder und sonstiges Drittmaterial unterliegen ebenfalls der genannten Creative Commons Lizenz, sofern sich aus der Abbildungslegende nichts anderes ergibt. Sofern das betreffende Material nicht unter der genannten Creative Commons Lizenz steht und die betreffende Handlung nicht nach gesetzlichen Vorschriften erlaubt ist, ist für die oben aufgeführten Weiterverwendungen des Materials die Einwilligung des jeweiligen Rechteinhabers einzuholen.

(c) (1) 